\title{
Review: psychoeducational programmes reduce MI recurrence and improve some physical health outcomes
}

\author{
Dusseldorp E, van Elderen T,Maes S, et al. A meta-analysis of psychoeducational programs for coronary heart disease \\ patients. Health Psychol 1999 Sep;18:506-19.
}

\author{
QUESTION: Can psychoeducational programmes, such as stress management and \\ health education, improve cardiac outcomes and physical health in patients with \\ coronary artery disease (CAD)?
}

\section{Data sources}

Studies were identified by searching Medline and PsycLIT (1974 to 1998) and by scanning bibliographies of relevant articles.

\section{Study selection}

Studies were selected if they involved patients who had had a cardiac event 6 months before treatment and if the study design was a randomised controlled trial (RCT) or quasi-randomised trial. Studies reporting effects on emotional distress were included only if effects on risk factors, related behaviours, morbidity, or cardiac mortality were also reported. Quasi-randomised trials were included only if samples were stratified or matched pairwise or if patients from the same hospital were allocated by using time periods.

\section{Data extraction}

2 reviewers independently extracted data on patients, interventions, setting, year of publication, study methods, and effect sizes for cardiac events and health outcomes. When reviewers disagreed, a third reviewer made the final decision.

\section{Main results}

37 studies (28 RCTs) met the inclusion criteria. Interventions were $<6$ weeks to $>6$ months in duration (mean $28 \mathrm{wk}$ ) and consisted of health education or stress management or both. Sometimes exercise training was used as well. Patients in the control group received standard care (30 studies), exercise training (6 studies), or health education (1 study). The combined results showed that psychoeducational programmes led to a decrease in MI recurrence, systolic blood pressure, total serum cholesterol level, weight, and smoking behaviour ( $\mathrm{p}<0.025$ for all comparisons) (table). The results were also generally positive, but not homogenous, for angina within 1 year of follow up (relative risk reduction [RRR] $18 \%, \mathrm{p}<0.025)$ and cardiac mortality after 2 years of follow up (RRR 34\%, $\mathrm{p}<0.025$ ). The groups did not differ for coronary artery bypass grafting, anxiety, or depression.

\section{Conclusion}

In patients with coronary artery disease, psychoeducational programmes help reduce myocardial infarction recurrence, long term cardiac mortality, short term angina, systolic blood pressure, total serum cholesterol level, weight, and smoking behaviour.

\section{COMMENTARY}

This meta-analysis by Dusseldorp and colleagues is consistent with previous studies showing that psychosocial interventions reduce cardiac mortality and cardiac events. ${ }^{1}$ It suggests that health education and stress management programmes in patients with CAD lead to a $34 \%$ reduction in long term cardiac mortality and a $29 \%$ reduction in recurrent myocardial infarction.

However, as the authors noted, this review has several limitations. Most studies gave only vague descriptions of the behavioural interventions. This makes it difficult for clinicians to determine which services are likely to benefit their patients. Most patients were men with a mean age in their 50s. Whether these programmes would provide the same benefit to higher risk patients, such as the elderly, is uncertain. It is also unclear whether these programmes would add to the benefits already achieved by drug therapy.

The authors included both quasi-experimental studies and RCTs, which could have weakened their conclusions, but they did adjust for pretest differences between the intervention and comparison groups. In addition, they found no difference in effect for random and non-random assignment. Therefore, the inclusion of only RCTs would probably not appreciably alter the reported results.

Only studies that showed reductions in risk factors (eg, blood pressure, cholesterol, body weight, and smoking) also showed a reduction in cardiac events or mortality. However, these risk factors were often poorly measured. For example, smoking behaviour was primarily measured by patient self report. Thus, a reported $63 \%$ reduction in smoking may be an overestimate. Nonetheless, patients with modifiable risk factors, especially tobacco use, may benefit most from psychoeducational interventions. Physicians should explore their patients' willingness to change lifestyle. Motivated patients should be encouraged to participate in these programmes.

Robert Gluckman, MD Providence-St. Vincent Medical Center Portland, Oregon, USA

1 Linden W, Stossel C, Maurice J. Psychosocial interventions for patients with coronary artery disease: a meta-analysis. Arch Intern Med 1996;156:745-52

Psychoeducational programmes $v$ standard care or other programmes for coronary artery disease* $^{*}$

\begin{tabular}{lll} 
Outcomes & $\begin{array}{l}\text { Number of } \\
\text { studies }\end{array}$ & $\begin{array}{l}\text { Weighted average } \\
\text { effect size } \mathrm{r}(95 \% \mathrm{CI})\end{array}$ \\
\hline MI recurrence at 1 to $10 \mathrm{y}$ & 16 & $0.03(0.01$ to 0.06$)$ \\
\hline Systolic BP decrease at $\{0.1$ to $2 \mathrm{y}\} \dagger$ & 8 & $0.12(0.06$ to 0.18$) \neq$ \\
\hline Serum cholesterol level decrease at $\{0.1$ to $2 \mathrm{y}\} \dagger$ & 7 & $0.25\{0.20$ to 0.30$\}+\ddagger$ \\
\hline Weight decrease at $\{0.1$ to $2 \mathrm{y}\}$ & 8 & $0.09(0.03$ to 0.15$) \neq$ \\
\hline Decrease in smoking at $\{0.1$ to $2 \mathrm{y}\} \dagger$ & 21 & $0.07(0.03$ to 0.10$)$ \\
\hline
\end{tabular}

$\mathrm{BP}=$ blood pressure; $\mathrm{MI}=$ myocardial infarction

fFollow up information and $\mathrm{Cl}$ supplied by author. EEffect sizes adjusted for pretest differences.
Netherlands

Organization for

Scientific Research.

For correspondence: Ms E Dusseldorp, Data Theory Group, Faculty of Social and Behavioral Sciences, Leiden University, $P O$ Box 9555,2300RB

Leiden, the

Netherlands. Fax +31

715273865 .
Source of funding: 